\title{
Follow-up observations of GW170817 with the MAGIC telescopes
}

\author{
A. Stamerra, ${ }^{a, e, j, *}$ O. S. Salafia, ${ }^{b, *}$ A. Berti, ${ }^{c, d}$ S. Covino, ${ }^{b}$ V. D'Elia, ${ }^{a, e}$ D. Miceli, ${ }^{g, i, *}$ L. \\ Nava, ${ }^{b}$ B. Patricelli, ${ }^{a, f}$ C. Righi, ${ }^{b, *}$ S. Inoue, ${ }^{g}$ L. A. Antonelli, ${ }^{a}$ S. Ansoldi, ${ }^{h}$ B. De \\ Lotto, $^{h}$ F. Longo ${ }^{h, i}$ and F. Tavecchio ${ }^{b}$ on behalf of the MAGIC Collaboration ${ }^{\dagger}$ \\ ${ }^{a}$ INAF - Osservatorio Astronomico di Roma, Rome, Italy \\ ${ }^{b}$ INAF - Osservatorio Astronomico di Brera, Merate, Italy \\ ${ }^{c}$ Istituto Nazionale Fisica Nucleare (INFN), Rome, Italy \\ ${ }^{d}$ also at Max-Planck-Institut für Physik, München, Germany \\ ${ }^{e}$ also at Space Science Data Center - Agenzia Spaziale Italiana, Rome, Italy \\ ${ }^{f}$ European Gravitational Observatory, Pisa, Italy \\ ${ }^{g}$ RIKEN, Wako, Saitama, Japan \\ ${ }^{h}$ Università di Udine and INFN Trieste, Udine, Italy \\ ${ }^{i}$ also at Dipartimento di Fisica, Università di Trieste, Trieste, Italy \\ ${ }^{j}$ also at Scuola Normale Superiore, Pisa, Italy \\ E-mail: antonio.stamerra@inaf.it, om.salafia@inaf.it (external \\ contributor), davide.miceli1993@gmail.com, chiara.righi@inaf.it
}

The discovery of the electromagnetic counterpart AT2017gfo and the GRB 170817A, associated to the binary neutron star merger GW170817, was one of the major advances in the study of gammaray bursts (GRBs) and the hallmark of the multi-messenger astronomy with gravitational waves. Another breakthrough in GRB physics is represented by the discovery of the highly energetic, teraelectronvolt (TeV) component in the GRB 190114C, possibly an universal component in all GRBs. This conclusion is also suggested by the hint of TeV emission in the short GRB 160821B and a few more events reported in the literature. The missing observational piece is the joint detection of $\mathrm{TeV}$ emission and gravitational waves from a short GRB and its progenitor. MAGIC observed the counterpart AT2017gfo as soon as the visibility conditions allowed it, namely from January to June 2018. These observations correspond to the maximum flux level observed in the radio and $\mathrm{X}$-ray bands. The upper limits derived from $\mathrm{TeV}$ observations are compared with the modelling of the late non-thermal emission using the multi-frequency SED.

$37^{\text {th }}$ International Cosmic Ray Conference (ICRC 2021)

July 12 th - 23rd, 2021

Online - Berlin, Germany

\footnotetext{
${ }^{*}$ Presenter

${ }^{\dagger}$ a complete list of the MAGIC Collaboration authors can be found at the end of the proceedings
} 


\section{Introduction}

The first detection of gravitational waves (GWs) from the coalescence of binary systems formed by two stellar mass black holes, GW150914 [1], commenced the era of the GW astronomy. The successive joint GW and electromagnetic (EM) observations from the binary neutron star (BNS) merger GW170817 [2-4] set the beginning of the multi-messenger astronomy with GWs. GW170817 was observed by Advanced LIGO [5] and Advanced Virgo [6] and was associated to the short Gamma-ray Burst GRB 170817A detected by Fermi-GBM [7] and INTEGRAL [8]. The joint GW-EM detection provided the first strong evidence of the association between (at least a fraction of) BNS and short-GRBs, envisaged by theoretical descriptions of the BNS coalescence [9] and by observational clues on short-GRB [10].

The prediction of the isotropic optical and near-infrared source powered by the decay of radioactive nuclei generated by r-process nucleosynthesis in the ejecta formed after the coalescence, was also confirmed with the discovery of the optical/IR counterpart AT 2017gfo, the long-sought kilonova, hosted in the galaxy NGC 4993 placed at a distance of $40 \mathrm{Mpc}$.

Interestingly an X-ray and radio counterpart did emerge in the days after the burst, later identified as the GRB afterglow non-thermal emission [11] (also [12] and references therein). The late increasing afterglow emission is expected by the interaction of a off-axis jet (whose axis is not aligned by several degrees with the line of sight) with the surrounding medium. In fact, the strongly beamed emission prevents the detection at off-axis angles until the bulk Lorentz factor, $\Gamma(t)$, of the collimated outflow is reduced by the deceleration of the jet; during the deceleration, the beaming cone angle $(\sim 1 / \Gamma(t))$ widens, eventually including the observer. The net effect is a delayed emission, whose intensity and delay depends on the off-axis angle. Successive radio interferometry observations did confirm the interpretation of the radio to X-ray emission as originated by an offaxis, structured jet with a viewing angle of $\approx 30 \mathrm{deg}[11,13,14]$. The radio and $\mathrm{X}$-ray emission did increase in the weeks following the merger, reaching a maximum after $\sim 155$ days post-merger, as visible in figure 1. The measurement of a superluminal motion in the radio [14, 32] confirmed the emergence of a narrow and relativistic jet, further supporting the off-axis scenario.

Up to today, GW170817 is the only GW event detected by Advanced LIGO and Advanced Virgo with EM counterparts, despite the several successive GW detections, mostly from binary black-hole mergers candidates [15, 16] (see, however, [17]).

GRBs afterglows are also known to emit very-high energy (VHE, $>100 \mathrm{GeV}$ ) gamma-rays, as shown by the observations of the long-GRBs GRB 190114C and the short-GRB 160821B by MAGIC [18, 19] and GRB 180720B and GRB 190829a by H.E.S.S. [20, 23]. The GeV-TeV emission from GRB 190114C clearly points to a new energetic component [25], predicted by a few models, and for which only observational, not conclusive, clues were available [33]. Interestingly, a single synchrotron component from the $\mathrm{X}$-ray to $\mathrm{TeV}$ has been suggestion to explain the $\mathrm{TeV}$ emission from GRB 190829A (see [23], but also [24]). A strong hint of VHE emission has been seen by MAGIC on the short-GRB 160821B [19], providing a compelling clue on the detectability of TeV emission from GW counterparts from compact object mergers. A follow-up of GW170817 has been performed by H.E.S.S., but no VHE counterpart was found, neither immediately after the burst [21], nor in late observations [22].

In this work we describe the follow-up campaign performed by the two 17-m MAGIC Cherenkov 


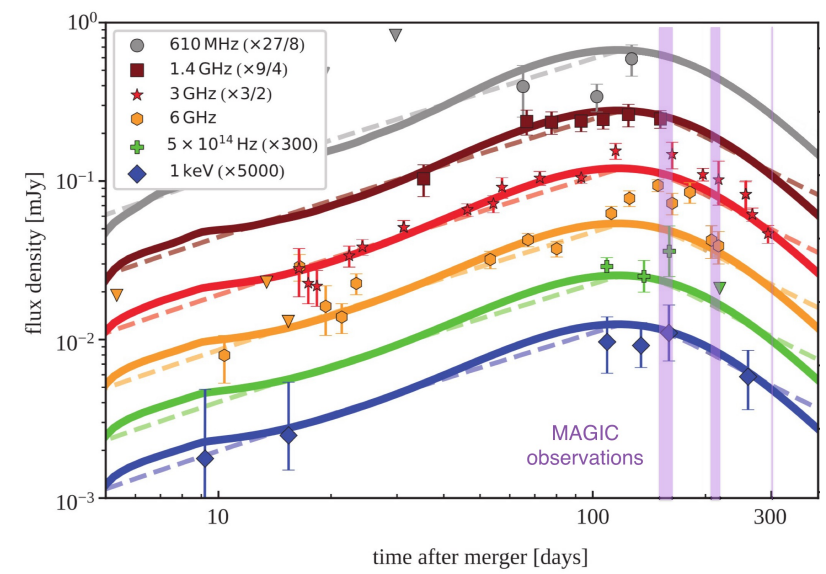

Figure 1: Radio, optical and X-ray light curves of the afterglow emission of GW 170817. Shaded violet vertical bars mark the times of the MAGIC observations. Figure is adapted from [14], see references therein for the sources of the data points. Solid lines show their best-fitting structured jet model, while dashed lines show a choked jet cocoon model.

telescopes in the months following the detection of GW170817 (Sec. 2) and the interpretation of the multi-wavelength (MWL) SED, in view of the numerous MWL observations performed from January to June 2018 (Sec. 3 and 4). The conclusions show that the capability of TeV measurements in constraining the physical parameters of the off-axis GRB model, depends strongly on the viewing angle and on the density of the merger environment.

\section{MAGIC follow-up observations of GW170817}

Starting from mid-December 2017/January 2018, the sky region hosting the optical counterpart associated to the GW170817, became visible to the MAGIC Cherenkov telescopes (located on the Canary Island of La Palma, Spain, Long.: -17.88, Lat.:+28.7) at zenith angles between 50 and $\sim 60 \mathrm{deg}$, corresponding to the maximum for the standard observing mode [26, 27].

MAGIC follow-up observations were performed for a total amount of $\sim 9.5 \mathrm{hrs}$ in 10 different nights from January to June 2018. The observing periods are shown in figure 1, superimposed to the radio and X-ray lightcurves of the afterglow of GW170817, extracted from [14]. The MAGIC data were taken in dark conditions, in absence of Moon, thus with a level of the night sky background (NSB) light relatively low. Only the data taken in good weather conditions, for which the transmission at a height of $9 \mathrm{~km}$ was above $85 \%$ were selected for the analysis. The estimation of the atmospheric transmission was provided by the LIDAR instrument, installed at the MAGIC site [28].

The standard data analysis chain with the MAGIC software package MARS [29] was used. No significant VHE emission was found. A set of upper limits (ULs) were evaluated in order to constrain the photon flux in the VHE band. The calculation was performed assuming a simple power law function for the intrinsic gamma-ray differential photon spectrum $d N / d E=E^{-\alpha}$ using the Rolke algorithm [30], with a confidence level (CL) of $95 \%$ and a total systematic uncertainty on the detection efficiency of 30\%. Three different photon indexes $\alpha=1.5,2.0,2.5$ were chosen. The absorption due to the extragalactic background light (EBL) model was taken into account as described in [31]-EBL model. The energy threshold of the event, calculated as the peak of the MC reconstructed energy distribution weighted for a spectrum with spectral index $\alpha=2$, was estimated to be $\sim 400 \mathrm{GeV}$. The resulting UL calculated for $E>400 \mathrm{GeV}$ is $3.6 \times 10^{-12} \mathrm{erg} \mathrm{cm}^{-2} \mathrm{~s}^{-1}$ for $\alpha=2.0$. This value varies within $15 \%$ when different slopes between 1.5 and 2.5 are considered. 


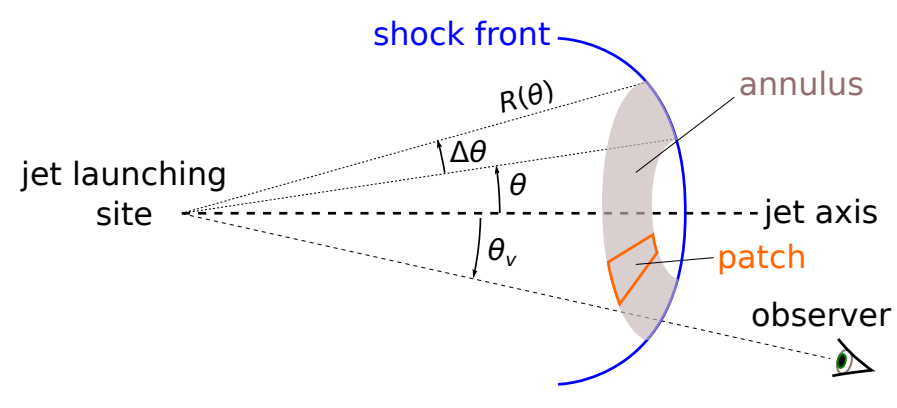

Figure 2: Geometry of the off-axis structured jet afterglow model. The jet is divided into thin annuli centered on the jet axis. In each annulus, the shock dynamics is computed from energy conservation. The annulus is then further divided into small azimuthal patches and the surface brightness of each patch is computed from the emission model, accounting for relativistic beaming.

\section{The multi-wavelength SED}

The description and modeling of the non-thermal emission originating in the narrow $\left(\theta_{\text {jet }} \sim\right.$ $3^{\circ}$ ), relativistic and energetic ( $E_{\text {isotropic,jet-core }} \sim 2 \times 10^{52} \mathrm{erg}$ ) jet, is done through the multiwavelength spectral energy distribution (SED), built using the radio, optical and X-ray data gathered in [37]. They are shown in Figure 3 as computed at 123 and 195 days post-merger. The interferometric measurements with VLBI provides the measurement of the displacement of the radio-core of the jet (the centroid of the radio emission)[14, 32], setting a limit on the viewing angle.

The spectrum of the X-ray emission measured by Chandra and XMM-Newton observatories is well described by a single power-law with photon index $\alpha=1.54_{-0.15}^{+0.19}$ consistent with the spectral index $\sim 1.57$ from radio to $\mathrm{X}$-ray. This points at a single power-law component from radio up to $\mathrm{X}$-rays, without an indication of a turnover up to $\sim 10 \mathrm{keV}$.

\section{The expected self-Compton emission and the TeV limits}

In order to compute the emission from the shock that arises as the structured jet sweeps the interstellar medium (ISM), we employ the same model as [14], described in detail in [34]. In brief, we divide the shock into thin annuli with a logarithmic grid in the polar angle, centered on the jet axis (see Fig. 2). In each annulus, we compute the shock dynamics from energy conservation: the jet isotropic-equivalent kinetic energy at angle $\theta$ is assumed to have the form

$$
E_{\mathrm{K}, \text { iso }}(\theta)=\frac{E_{\mathrm{c}}}{1+\left(\theta / \theta_{\mathrm{c}}\right)^{s_{1}}},
$$

while the jet Lorentz factor is

$$
\Gamma_{0}(\theta)=1+\frac{\Gamma_{\mathrm{c}}-1}{1+\left(\theta / \theta_{\mathrm{c}}\right)^{s_{2}}},
$$

where $\theta_{\mathrm{c}}$ is the half-opening angle of the jet core, and $E_{\mathrm{c}}, \Gamma_{\mathrm{c}}, s_{1}$ and $s_{2}$ are parameters. The isotropic-equivalent mass swept when the shock has reached a radius $R$ is $m(R)=4 \pi R^{3} n m_{\mathrm{p}} / 3$, where $n$ is the ISM number density and $m_{\mathrm{p}}$ is the proton mass. The shock dynamics, that is, the evolution of the shocked material Lorentz factor $\Gamma(\theta, R)$ as a function of the radius $R$ at each angle $\theta$, is obtained by enforcing the approximate energy conservation condition [35] $E_{\mathrm{K} \text {,iso }}(\theta)=$ $\Gamma(\theta, R) E_{\mathrm{K} \text {,iso }}(\theta) / \Gamma_{0}(\theta)+\Gamma^{2}(\theta, R) m(R) c^{2}$. We neglect lateral energy transport as it is unimportant 


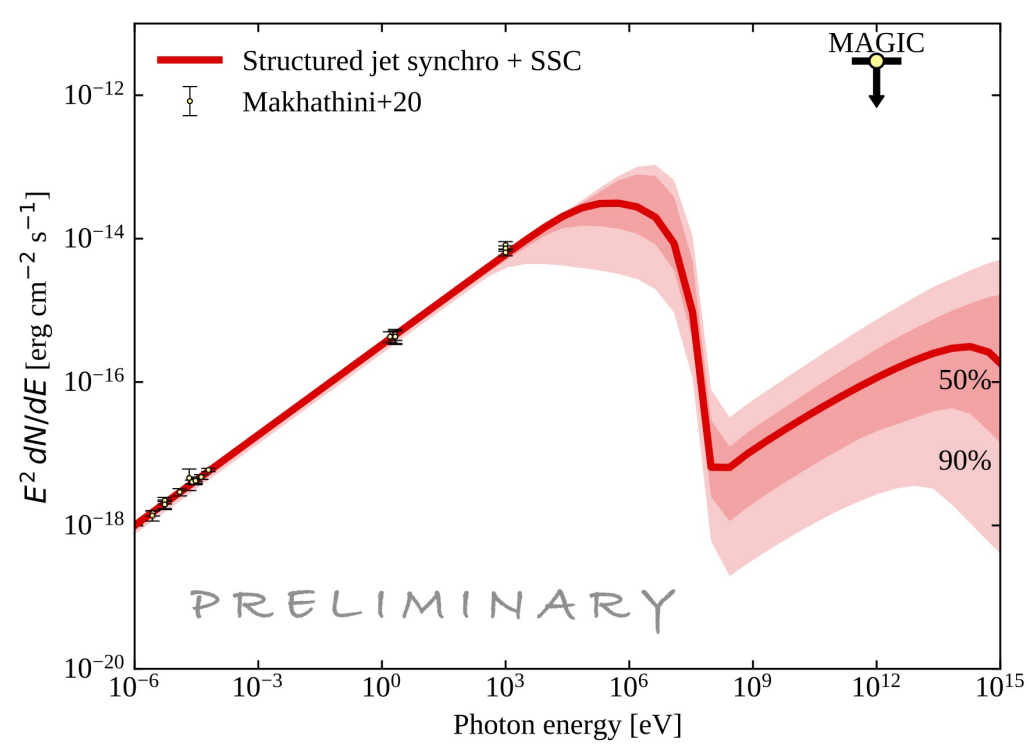

Figure 3: Expected Synchrotron and self-Compton emission at 155 days post-merger, using the best-fit parameters obtained from the radio, optical and X-ray light curves and the apparent displacement of the VLBI centroid, as measured in [14]. Small circles with error bars show GRB 170817A data [37] between 123 and 195 days ( \pm 0.1 dex from 155 days), while our upper limit is shown in the upper right corner of the plot.

at the times considered [36], so that we can consider annuli to evolve independently of each other. In each annulus, relativistic electrons are assumed to be injected in the shock downstream with a power law energy distribution $d n_{\mathrm{e}} / d \gamma \propto \gamma^{-p}$, where $\gamma$ is the electron Lorentz factor in the fluid comoving frame, due to in situ acceleration. Electrons are assumed to hold a constant fraction $\epsilon_{\mathrm{e}}$ of the available energy density in the shock downstream, while an effectively isotropic, smallscale-turbulence-amplified magnetic field is assumed to hold another fraction $\epsilon_{\mathrm{B}}$. Electron cooling is computed accounting for synchrotron and self-Compton losses, and the total emission is finally integrated over equal-arrival-time surfaces, accounting for relativistic beaming and for the off-axis viewing angle $\theta_{\mathrm{v}}$. In Figure 3 we show (red solid line) the predicted spectral energy distribution at 155 days post-merger, including synchrotron and self-Compton emission, using the best fit parameters obtained in [14] by fitting the model to the multi-wavelength data, including the radio VLBI centroid displacement. The light pink (resp. dark pink) band shows the 90\% (resp. 50\%) confidence band obtained by propagating the uncertainties on the parameters.

Figure 4 shows the magnetic field strength in the shock downstream at the same time, computed as a surface-brightness-weighted average over the equal-arrival-time surface, namely

$$
B=\frac{\int_{0}^{2 \pi} d \phi \int_{0}^{\pi / 2} \sin \theta d \theta I_{v}(\theta, \phi) B(\theta, \phi)}{\int_{0}^{2 \pi} d \phi \int_{0}^{\pi / 2} \sin \theta d \theta I_{v}(\theta, \phi)}
$$

where $I_{v}(\theta, \phi)$ is the surface brightness of the $(\theta, \phi)$ point of the equal-arrival-time surface at observer frequency $v$ and $B(\theta, \phi)$ is the downstream magnetic field at the corresponding position 
of the shock. We set $v=10^{14} \mathrm{~Hz}$ as this is where the typical seed photons of the inverse-Comptonscattered $1 \mathrm{TeV}$ photons lie. The result is $B(155 \mathrm{~d})=240_{-140}^{+590} \mu \mathrm{G}$, with $68 \%$ confidence error bars propagated from the model parameter uncertainties This can be compared with a simple analytical estimate from the standard afterglow model [38], which reads

$$
B \sim 130 \epsilon_{\mathrm{B},-4}^{1 / 2} n_{-4}^{5 / 8} E_{52}^{1 / 8} t_{\mathrm{obs}, 2}^{-3 / 8} \mu \mathrm{G}
$$

where $Q_{x}=10^{x} Q$ for any quantity $Q$, and all quantities are in cgs units except for the observer time $t_{\mathrm{obs}}$ which is expressed in days. The formula can be applied to the structured jet core (which dominates the emission at our times), as the jet is effectively on-axis after the lightcurve peak. With the best fit parameters from [14], this yields $B \sim 190 \mu \mathrm{G}$, in good agreement with the more refined estimate above.

\section{Conclusions}

MAGIC upper limits, and in general any measurement by the present generation of Cherenkov telescopes, are not sensitive enough to constrain the expected synchrotron self-Compton (SSC) emission predicted in this work, even when considering the parameter uncertainties (as shown by the shaded area in Figure 3). We note that the model parameters we use here were derived by fitting the emission model (which is based on detailed physical modelling of the shock dynamics and of its emission) to the available multi-wavelength light curves, and to the radio-centroid displacement observed with VLBI. While an attempt at modelling a SED with an SSC model at a single time might result in different fit parameters with a higher $\mathrm{TeV}$ emission, as in [22], this would not be consistent with the observed evolution and would not provide a physically viable description of the late afterglow of GRB 170817.

While this might seem daunting for what concerns the prospects for VHE detection of electromagnetic counterparts to GW sources, we note that some particular conditions in this source were not particularly favourable, such as the low interstellar medium density $n \lesssim 10^{-4} \mathrm{~cm}^{-3}$ and the relatively large viewing angle $\theta_{\mathrm{v}} \sim 15-25 \mathrm{deg}$. A smaller viewing angle would have anticipated the time at which the light curve would have resembled that of an on-axis jet, leading to a larger peak luminosity; a larger interstellar medium density (the GW170817 one was low when compared to the distribution of SGRBs, see e.g. [39]) would make the emission brighter in general (apart from low radio frequencies, where synchrotron self-absorption may come into play). As an example, we show in Figure 5 light curves at $1 \mathrm{TeV}$ for a jet with the same parameters as that of GW170817, but placed in a slightly denser interstellar medium with $n=5 \times 10^{-2} \mathrm{~cm}^{-3}$, which corresponds to the median of the short GRB sample of [39]. Different colours show different viewing angles. For a similar sensitivity as that reached in our observations described above, such a jet would be detectable at peak for viewing angles $\theta_{\text {view }} \lesssim 10 \mathrm{deg}$, while next-generation facilities would be able to detect the emission at larger viewing angles.

A possibly even more favourable scenario would be represented by the presence of a significant interaction between the relativistic electrons in the structured jet forward shock with an external radiation field, e.g. the optical/IR emission of the kilonova [40]. This would further enhance the resulting inverse Compton emission. 

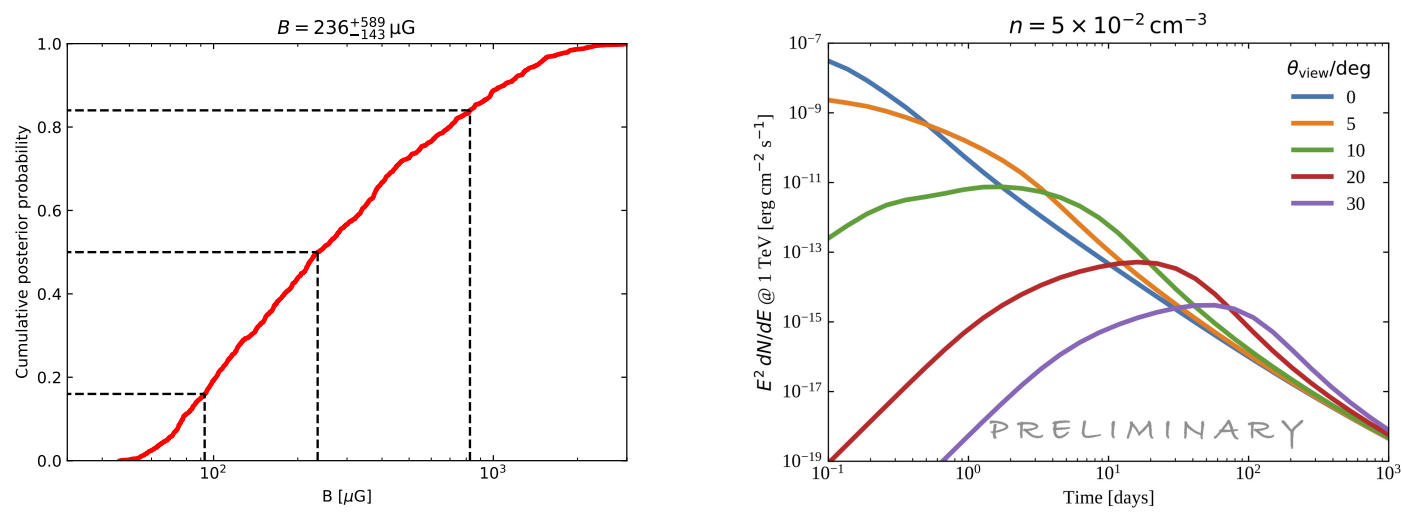

Figure 4: Cumulative posterior probability density of Figure 5: Light curves at $1 \mathrm{TeV}$ for a GW170817-like the downstream magnetic field. The red line shows jet under more favourable conditions. For the comthe cumulative posterior probability density of the putation we assumed the best fit jet parameters from forward shock downstream magnetic field (averaged [14], but we placed the jet in a slighty denser interover the surface brightness of the shock) at 155 days stellar medium $\left(n=5 \times 10^{-2} \mathrm{~cm}^{-3}\right)$ and we varied the post-merger according to our model. Black dashed viewing angle (color-coded as shown in the legend). lines mark the positions of the $16^{\text {th }}, 50^{\text {th }}$ and $84^{\text {th }}$ percentiles, that yield the one-sigma confidence result shown on top of the plot.

\section{Acknowledgments}

We acknowledge the support from the agencies and organizations listed here: https:// magic . mpp .mpg . de/acknowledgments_ICRC2021

\section{References}

[1] Abbott, B.P., Abbott, R., Abbott, T.D., et al. 2016, Phys. Rev. Lett. 116, 061102

[2] Abbott, B.P., Abbott, R., Abbott, T.D., et al. 2017, Phys. Rev. Lett. 119, 161101

[3] Abbott, B.P., Abbott, R., Abbott, T.D., et al. 2017, Astrophys. J. Lett. 848, 12

[4] Abbott, B.P., Abbott, R., Abbott, T.D., et al. 2017, Astrophys. J. Lett. 848, 13

[5] Harry, G.M., and LIGO Scientific Collab., Classical and Quantum Gravity 27, 084006 (2010)

[6] Acernese, F., et al. Classical and Quantum Gravity 32, 024001 (2015)

[7] Goldstein, A. et al. 2017, Astrophys. J. Lett. 848, 14

[8] Savchenko, V. et al. 2017, Astrophys. J. Lett. 848, 15

[9] Metzger B.D., Berger, E. 2012, ApJ, 746, 48

[10] Narayan, R.; Paczynski, B.; Piran, T. 1992, Astrophys. J. L., 395, L83-L86

[11] Troja E. et al. 2017, Nature, 551, 71 
[12] Abbott et al. 2017, Astrophys. J. Lett. 848, 12

[13] Pian E. 2017, Nature, 551, 67

[14] Ghirlanda, G. and Salafia, O. S. et al. 2019, Science, 363, 968

[15] Abbott, B.P., Abbott, R., Abbott, T.D., et al. 2019, PRX, 9, 031040

[16] Abbott, B.P., Abbott, R., Abbott, T.D., et al. 2020, arXiv:2010.14527

[17] Graham, M.J., Ford, K.E.S., McKernan, B., et al. 2020, PRL, 124, 251102

[18] MAGIC collaboration 2019, Nature, 575, 455

[19] Acciari, V.A. et al. 2021, ApJ, 908, 90

[20] Abdalla, H. et al. 2019, Nature, 575, 464

[21] Abdalla, H. et al. 2017, Astrophys. J. Lett. 85022

[22] Abdalla, H. et al. 2020, Astrophys. J. Lett. 894, 16

[23] H.E.S.S. Collaboration et al. 2021, Science 372, 1081-1085

[24] Salafia, O., 2021, arXiv:2106.07169

[25] MAGIC collaboration, et al. 2019, Nature, 575, 459

[26] Aleksic et al. 2016, Astrop. Phys., 72, 76

[27] MAGIC collaboration 2020, A\&A, 635, 158

[28] Fruck, C. et al. 2014, arXiv:1403.3591

[29] Zanin, R., et al. 2013, in Proceedings of the 33rd ICRC, 0773

[30] Rolke, W. A. et al. 2005, Nucl. Instr. Meth. Phys. Res. A, 551, 493

[31] Dominguez A., et al. 2011, MNRAS, 410, 4

[32] Mooley, K. P., Deller, A. T., Gottlieb, O., et al. 2018a, Nature, 561, 355

[33] Nava, L. 2018 International Journal of Modern Physics D, 27

[34] Salafia, O. et al. 2019, A\&A, 628, 18

[35] Panaitescu, A. \& Kumar, P. 2000, ApJL, 560, L49

[36] Kumar, P. \& Granot 2003,

[37] Makhathini S., Mooley K. P., et al. 2020, arXiv, arXiv:2006.02382

[38] Sari R., Piran T., Narayan R., 1998, ApJL, 497, L17. doi:10.1086/311269

[39] Fong W., Berger E., Margutti R., Zauderer B. A., 2015, ApJ, 815, 102.

[40] Linial I., Sari R., 2019, MNRAS, 483, 624. doi:10.1093/mnras/sty3170 


\section{The MAGIC Collaboration}

V. A. Acciari ${ }^{1}$, S. Ansoldi ${ }^{2,41}$, L. A. Antonelli ${ }^{3}$, A. Arbet Engels $^{4}$, M. Artero ${ }^{5}$, K. Asano ${ }^{6}$, D. Baack ${ }^{7}$, A. Babić ${ }^{8}$, A. Baquero ${ }^{9}$, U. Barres de Almeida ${ }^{10}$, J. A. Barrio ${ }^{9}$, I. Batković ${ }^{11}$, J. Becerra González ${ }^{1}$, W. Bednarek ${ }^{12}$, L. Bellizzi ${ }^{13}$, E. Bernardini ${ }^{14}$, M. Bernardos ${ }^{11}$, A. Berti ${ }^{15}$, J. Besenrieder ${ }^{15}$, W. Bhattacharyya ${ }^{14}$, C. Bigongiari ${ }^{3}$, A. Biland ${ }^{4}$, O. Blanch ${ }^{5}$, H. Bökenkamp ${ }^{7}$, G. Bonnoli ${ }^{16}$, Ž. Bošnjak $^{8}$, G. Busetto ${ }^{11}$, R. Carosi ${ }^{17}$, G. Ceribella ${ }^{15}$, M. Cerruti ${ }^{18}$, Y. Chai ${ }^{15}$, A. Chilingarian ${ }^{19}$, S. Cikota $^{8}$, S. M. Colak ${ }^{5}$, E. Colombo ${ }^{1}$, J. L. Contreras ${ }^{9}$, J. Cortina ${ }^{20}$, S. Covino ${ }^{3}$, G. D’Amico ${ }^{15,42}$, V. D’Elia ${ }^{3}$, P. Da Vela ${ }^{17,43}$, F. Dazzi ${ }^{3}$, A. De Angelis ${ }^{11}$, B. De Lotto ${ }^{2}$, M. Delfino ${ }^{5,44}$, J. Delgado ${ }^{5,44}$, C. Delgado Mendez ${ }^{20}$, D. Depaoli ${ }^{21}$, F. Di Pierro ${ }^{21}$, L. Di Venere ${ }^{22}$, E. Do Souto Espiñeira ${ }^{5}$, D. Dominis Prester $^{23}$, A. Donini ${ }^{2}$, D. Dorner ${ }^{24}$, M. Doro ${ }^{11}$, D. Elsaesser ${ }^{7}$, V. Fallah Ramazani ${ }^{25,45}$, A. Fattorini ${ }^{7}$, M. V. Fonseca ${ }^{9}$, L. Font ${ }^{26}$, C. Fruck ${ }^{15}$, S. Fukami ${ }^{6}$, Y. Fukazawa ${ }^{27}$, R. J. García López ${ }^{1}$, M. Garczarczyk ${ }^{14}$, S. Gasparyan ${ }^{28}$, M. Gaug ${ }^{26}$, N. Giglietto ${ }^{22}$, F. Giordano ${ }^{22}$, P. Gliwny ${ }^{12}$, N. Godinović ${ }^{29}$, J. G. Green ${ }^{3}$, D. Green ${ }^{15}$, D. Hadasch ${ }^{6}$, A. Hahn ${ }^{15}$, L. Heckmann ${ }^{15}$, J. Herrera ${ }^{1}$, J. Hoang ${ }^{9,46}$, D. Hrupec ${ }^{30}$, M. Hütten ${ }^{15}$, T. Inada ${ }^{6}$, K. Ishio ${ }^{12}$, Y. Iwamura ${ }^{6}$, I. Jiménez Martínez ${ }^{20}$, J. Jormanainen ${ }^{25}$, L. Jouvin ${ }^{5}$, M. Karjalainen ${ }^{1}$, D. Kerszberg ${ }^{5}$, Y. Kobayashi ${ }^{6}$, H. Kubo ${ }^{31}$, J. Kushida ${ }^{32}$, A. Lamastra ${ }^{3}$, D. Lelas ${ }^{29}$, F. Leone ${ }^{3}$, E. Lindfors ${ }^{25}$, L. Linhoff ${ }^{7}$, S. Lombardi ${ }^{3}$, F. Longo ${ }^{2,47}$, R. López-Coto ${ }^{11}$, M. López-Moya ${ }^{9}$, A. López-Oramas ${ }^{1}$, S. Loporchio ${ }^{22}$, B. Machado de Oliveira Fraga $^{10}$, C. Maggio ${ }^{26}$, P. Majumdar ${ }^{33}$, M. Makariev ${ }^{34}$, M. Mallamaci ${ }^{11}$, G. Maneva ${ }^{34}$, M. Manganaro ${ }^{23}$, K. Mannheim ${ }^{24}$, L. Maraschi ${ }^{3}$, M. Mariotti ${ }^{11}$, M. Martínez ${ }^{5}$, D. Mazin ${ }^{6,15}$, S. Menchiari ${ }^{13}$, S. Mender ${ }^{7}$, S. Mićanović ${ }^{23}$, D. Miceli ${ }^{2,49}$, T. Miener $^{9}$, J. M. Miranda ${ }^{13}$, R. Mirzoyan ${ }^{15}$, E. Molina ${ }^{18}$, A. Moralejo ${ }^{5}$, D. Morcuende ${ }^{9}$, V. Moreno ${ }^{26}$, E. Moretti ${ }^{5}$, T. Nakamori ${ }^{35}$, L. Nava ${ }^{3}$, V. Neustroev ${ }^{36}$, C. Nigro $^{5}$, K. Nilsson ${ }^{25}$, K. Nishijima ${ }^{32}$, K. Noda $^{6}$, S. Nozaki ${ }^{31}$, Y. Ohtani' ${ }^{6}$, T. Oka ${ }^{31}$, J. Otero-Santos ${ }^{1}$, S. Paiano ${ }^{3}$, M. Palatiello ${ }^{2}$, D. Paneque ${ }^{15}$, R. Paoletti ${ }^{13}$, J. M. Paredes ${ }^{18}$, L. Pavletić ${ }^{23}$, P. Peñil ${ }^{9}$, M. Persic ${ }^{2,50}$, M. Pihet ${ }^{15}$, P. G. Prada Moroni ${ }^{17}$, E. Prandini ${ }^{11}$, C. Priyadarshi ${ }^{5}$, I. Puljak ${ }^{29}$, W. Rhode ${ }^{7}$, M. Ribó ${ }^{18}$, J. Rico ${ }^{5}$, C. Righi ${ }^{3}$, A. Rugliancich ${ }^{17}$, N. Sahakyan ${ }^{28}$, T. Saito ${ }^{6}$, S. Sakurai ${ }^{6}$, K. Satalecka ${ }^{14}$, F. G. Saturni ${ }^{3}$, B. Schleicher ${ }^{24}$, K. Schmidt ${ }^{7}$, T. Schweizer ${ }^{15}$, J. Sitarek ${ }^{12}$, I. Šnidaric ${ }^{37}$, D. Sobczynska ${ }^{12}$, A. Spolon ${ }^{11}$, A. Stamerra ${ }^{3}$, J. Strišković ${ }^{30}$, D. Strom ${ }^{15}$, M. Strzys ${ }^{6}$, Y. Suda ${ }^{27}$, T. Surić ${ }^{37}$, M. Takahashi ${ }^{6}$, R. Takeishi ${ }^{6}$, F. Tavecchio ${ }^{3}$, P. Temnikov ${ }^{34}$, T. Terzić ${ }^{23}$, M. Teshima ${ }^{15,6}$, L. Tosti ${ }^{38}$, S. Truzzi ${ }^{13}$, A. Tutone ${ }^{3}$, S. Ubach ${ }^{26}$, J. van Scherpenberg ${ }^{15}$, G. Vanzo ${ }^{1}$, M. Vazquez Acosta ${ }^{1}$, S. Ventura ${ }^{13}$, V. Verguilov ${ }^{34}$, C. F. Vigorito ${ }^{21}$, V. Vitale ${ }^{39}$, I. Vovk $^{6}$, M. Will ${ }^{15}$, C. Wunderlich ${ }^{13}$, T. Yamamoto $^{40}$, and D. Zarić 29

${ }^{1}$ Instituto de Astrofísica de Canarias and Dpto. de Astrofísica, Universidad de La Laguna, E-38200, La Laguna, Tenerife, Spain ${ }^{2}$ Università di Udine and INFN Trieste, I-33100 Udine, Italy ${ }^{3}$ National Institute for Astrophysics (INAF), I-00136 Rome, Italy ${ }^{4}$ ETH Zürich, CH-8093 Zürich, Switzerland ${ }^{5}$ Institut de Física d'Altes Energies (IFAE), The Barcelona Institute of Science and Technology (BIST), E-08193 Bellaterra (Barcelona), Spain ${ }^{6}$ Japanese MAGIC Group: Institute for Cosmic Ray Research (ICRR), The University of Tokyo, Kashiwa, 277-8582 Chiba, Japan ${ }^{7}$ Technische Universität Dortmund, D-44221 Dortmund, Germany ${ }^{8}$ Croatian MAGIC Group: University of Zagreb, Faculty of Electrical Engineering and Computing (FER), 10000 Zagreb, Croatia ${ }^{9}$ IPARCOS Institute and EMFTEL Department, Universidad Complutense de Madrid, E-28040 Madrid, Spain ${ }^{10}$ Centro Brasileiro de Pesquisas Físicas (CBPF), 22290-180 URCA, Rio de Janeiro (RJ), Brazil ${ }^{11}$ Università di Padova and INFN, I-35131 Padova, Italy ${ }^{12}$ University of Lodz, Faculty of Physics and Applied Informatics, Department of Astrophysics, 90-236 Lodz, Poland ${ }^{13}$ Università di Siena and INFN Pisa, I-53100 Siena, Italy ${ }^{14}$ Deutsches Elektronen-Synchrotron (DESY), D-15738 Zeuthen, Germany ${ }^{15}$ Max-Planck-Institut für Physik, D-80805 München, Germany ${ }^{16}$ Instituto de Astrofísica de Andalucía-CSIC, Glorieta de la Astronomía s/n, 18008, Granada, Spain ${ }^{17}$ Università di Pisa and INFN Pisa, I-56126 Pisa, Italy ${ }^{18}$ Universitat de Barcelona, ICCUB, IEEC-UB, E-08028 Barcelona, Spain ${ }^{19}$ Armenian MAGIC Group: A. Alikhanyan National Science Laboratory, 0036 Yerevan, Armenia ${ }^{20}$ Centro de Investigaciones Energéticas, Medioambientales y Tecnológicas, E-28040 Madrid, Spain ${ }^{21}$ INFN MAGIC Group: INFN Sezione di Torino and Università degli Studi di Torino, I-10125 Torino, Italy 22 INFN MAGIC Group: INFN Sezione di Bari and Dipartimento Interateneo di Fisica dell'Università e del Politecnico di Bari, I-70125 Bari, Italy ${ }^{23}$ Croatian MAGIC Group: University of Rijeka, Department of Physics, 51000 Rijeka, Croatia ${ }^{24}$ Universität Würzburg, D-97074 Würzburg, Germany ${ }^{25}$ Finnish MAGIC Group: Finnish Centre for Astronomy with ESO, University of Turku, FI-20014 Turku, Finland ${ }^{26}$ Departament de Física, and CERES-IEEC, Universitat Autònoma de Barcelona, E-08193 Bellaterra, Spain 27 Japanese MAGIC Group: Physics Program, Graduate School of Advanced Science and Engineering, Hiroshima University, 739-8526 Hiroshima, Japan ${ }^{28}$ Armenian MAGIC Group: ICRANet-Armenia at NAS RA, 0019 Yerevan, Armenia ${ }^{29}$ Croatian MAGIC Group: University of Split, Faculty of Electrical Engineering, Mechanical Engineering and Naval Architecture (FESB), 21000 Split, Croatia ${ }^{30}$ Croatian MAGIC Group: Josip Juraj Strossmayer University of Osijek, Department of Physics, 31000 Osijek, Croatia ${ }^{31}$ Japanese MAGIC Group: Department of Physics, Kyoto University, 606-8502 Kyoto, Japan ${ }^{32}$ Japanese MAGIC Group: Department of Physics, Tokai University, Hiratsuka, 259-1292 Kanagawa, Japan ${ }^{33}$ Saha Institute of Nuclear Physics, HBNI, 1/AF Bidhannagar, Salt Lake, Sector-1, Kolkata 700064, India ${ }^{34}$ Inst. for Nucl. Research and Nucl. Energy, Bulgarian Academy of Sciences, BG-1784 Sofia, Bulgaria ${ }^{35}$ Japanese MAGIC Group: Department of Physics, Yamagata University, Yamagata 990-8560, Japan ${ }^{36}$ Finnish MAGIC Group: Astronomy Research Unit, University of Oulu, FI-90014 Oulu, Finland ${ }^{37}$ Croatian MAGIC Group: Ruđer Bošković Institute, 10000 Zagreb, Croatia ${ }^{38}$ INFN MAGIC Group: INFN Sezione di Perugia, I-06123 Perugia, Italy ${ }^{39}$ INFN MAGIC Group: INFN Roma Tor Vergata, I-00133 Roma, Italy ${ }^{40}$ Japanese MAGIC Group: Department of Physics, Konan University, Kobe, Hyogo 6588501, Japan ${ }^{41}$ also at International Center for Relativistic Astrophysics (ICRA), Rome, Italy ${ }^{42}$ now at Department for Physics and Technology, University of Bergen, NO-5020, Norway ${ }^{43}$ now at University of Innsbruck ${ }^{44}$ also at Port d'Informació Científica (PIC), E-08193 Bellaterra (Barcelona), Spain ${ }^{45}$ now at Ruhr-Universität Bochum, Fakultät für Physik und Astronomie, Astronomisches Institut (AIRUB), 44801 Bochum, Germany ${ }^{46}$ now at Department of Astronomy, University of California Berkeley, Berkeley CA $94720{ }^{47}$ also at Dipartimento di Fisica, Università di Trieste, I-34127 Trieste, Italy 49 now at Laboratoire d'Annecy de Physique des Particules (LAPP), CNRS-IN2P3, 74941 Annecy Cedex, France ${ }^{50}$ also at INAF Trieste and Dept. of Physics and Astronomy, University of 
Bologna, Bologna, Italy 